\title{
Spike timing regularity depends on the firing time scale under propofol-induced anesthetic condition
}

\author{
Mie Kajiwara ${ }^{1}$, Risako Kato ${ }^{2}$, Yoshiyuki Oi ${ }^{1}$, Masayuki Kobayashi ${ }^{2}$ \\ ${ }^{I}$ Department of Anesthesiology, Nihon University School of Dentistry, Japan, ${ }^{2}$ Department of Pharmacology, Nihon \\ University School of Dentistry, Japan
}

Propofol is widely used for general anesthesia and sedation in various medical procedures. Propofol produces unconsciousness by potentiating $\gamma$-aminobutyric acid type $\mathrm{A}\left(\mathrm{GABA}_{\mathrm{A}}\right)$ receptor-mediated inhibitory synaptic currents, however, little is known how propofol modulates the activities of the cortical local circuits in vivo. We performed extracellular unit recording in the rat insular cortex under awake and anesthetic conditions. Spike firing was transformed by unfolding transformation based on random matrix theory. This procedure can find out whether the neuronal firing with correlation in the short or long time scale in normalized histogram by existence of repulsion and decay pattern, without effects of neuronal firing properties. We classified neurons with high spontaneous firing frequency $(>5 \mathrm{~Hz})$ and bursting as HFB neurons $(n=9)$, and the others with low spontaneous firing frequency and without bursting as non-HFB neurons $(\mathrm{n}=91)$. Most of HFB neurons have shorter spike width than non-HFB neurons. Systemic injection of propofol (12 $\mathrm{mg} / \mathrm{kg}$ ) reduced firing frequency in all HFB neurons and $88 \%$ of non-HFB neurons. Unfolding transformation demonstrated that both HFB and non-HFB neurons showed a decrease in the number of neurons with repulsion by propofol application. In other words, propofol makes the correlation between a spike and subsequent spikes in short time scale weakened in both types of neurons. In 56\% of HFB and 44\% of non-HFB neurons under awake conditions, the decay phases of normalized histograms of spontaneous firing were fitted to an exponential function, indicating that the first spike had less correlation with subsequent spikes in the long time scale. In contrast, under propofol-induced anesthesia conditions, the number of non-HFB neurons fitted to an exponential function increased to 64\%, but there was little change in HFB neurons. These results suggest that regularity of spike timing is maintained in HFB neurons rather than in non-HFB neurons after injection of propofol. These findings may reflect the $\mathrm{GABA}_{\mathrm{A}}$ receptor-mediated regulation of cortical activities. 